\title{
Adult Mesenchymal Hamartoma of the Liver: Case Report and Literature Review
}

\author{
Zachary Klaassen $^{\text {a, b }}$ Prakash R. Paragi ${ }^{a}$ \\ Ronald S. Chamberlain ${ }^{\mathrm{a}-\mathrm{c}}$ \\ aDepartment of Surgery, Saint Barnabas Medical Center, Livingston, N.J., USA; \\ bSt. George's University School of Medicine, St. George's, Grenada, West Indies; \\ 'Department of Surgery, University of Medicine and Dentistry of New Jersey, \\ Newark, N.J., USA
}

\section{Key Words}

Mesenchymal hamartoma · Hepatobiliary surgery · Hepatic mass · Undifferentiated embryonal sarcoma

\begin{abstract}
Mesenchymal hamartoma of the liver $(\mathrm{MHL})$ is a rare benign lesion occurring primarily in the pediatric population. While the precise pathogenesis of the tumor is not certain, the most common theory relates to aberrant mesenchyme development in the portal tract likely related to the bile ducts. A 53-year-old female was evaluated for an incidental liver mass. Initial CT scan showed a cystic lesion in the right lobe of the liver and follow-up imaging revealed an increase in size and the percent solid component within the mass. In view of these changes, a nondiagnostic biopsy was obtained followed by extirpation of the lesion. Gross pathological review of the lesion identified a $9 \times 9 \times 7.5 \mathrm{~cm}$, pink-yellow-tan, gelatinous mass, with a $>1 \mathrm{~cm}$ clear surgical margin. Histologically, the mass consisted of benign dilated bile ducts, as well as myxoid stroma with spindle cells showing smooth muscle differentiation. The patient was discharged home on postoperative day five. A review of the literature for MHL in adults reports 30 previous cases, predominantly published as individual case reports describing the size, lobe(s) of the liver affected, and the cystic/solid nature of the tumor. MHL in adults may represent a potentially premalignant lesion, as the emerging literature supports a potential relationship between $\mathrm{MHL}$ and malignant undifferentiated embryonal sarcoma in regards to cytogenetic analysis. Aggressive surgical management of MHL in adults is mandated when feasible.
\end{abstract}




\section{Introduction}

Mesenchymal hamartoma of the liver (MHL) is a benign lesion that classically presents as an enlarging abdominal mass in children before the age of two. Maresch initially described the tumor in 1903, but the term MHL was first used by Edmondson in 1956 [1]. MHL may affect either lobe of the liver and presents as a cystic or solid mass, and on occasion both components may be present. Alkaline phosphatase, $\beta$-human chorionic gonadotropin, serum transaminases and $\alpha$-fetoprotein are usually within normal limits in patients with MHL. Hematoxylin and eosin staining as well as immunohistochemical studies $[2,3]$ have described MHL as having spindle cells positive for vimentin and smooth muscle actin and negative for CD31, CD34 and S100 proteins, while the ducts stain positive for cytokeratin 7 and negative for cytokeratin 20. Even though the exact pathogenesis of MHL is largely unknown, the most common theory is that it represents aberrant primitive mesenchyme development in the portal tract likely relating to the bile ducts.

\section{Case Report}

A 53-year-old white female presented in January 2007 with colicky abdominal pain secondary to ureteral stones; subsequent abdominal ultrasound imaging and a CT scan of the abdomen demonstrated an incidental liver mass (fig. 1). This lesion was initially thought to be a hemangioma, however follow-up imaging demonstrated an increase in the size of the mass, notably the solid component. This change in size as well as the characteristics of the mass prompted percutaneous biopsy in May 2008, which was nondiagnostic. The patient was referred for a hepatobiliary surgical consult. The patient's past surgical history was noncontributory, consisting of cyst removal of the left breast at the age of 16, a partial thyroidectomy in 2001 and a total abdominal hysterectomy with partial oophorectomy in 2006.

The preoperative work-up included a CEA level of $<0.5 \mathrm{ng} / \mathrm{ml}$, an $\alpha$-fetoprotein level of $3.6 \mathrm{ng} / \mathrm{ml}$, a CA 19-9 level of $17 \mathrm{U} / \mathrm{ml}$, a negative hepatitis panel, a total bilirubin level of $0.3 \mathrm{mg} / \mathrm{dl}$, an alkaline phosphatase level of $87 \mathrm{U} / \mathrm{l}$, an aspartate transaminase level of $45 \mathrm{U} / \mathrm{l}$ and an alanine transaminase level of $50 \mathrm{U} / \mathrm{l}$. Repeat abdominal CT scan (ig. 2) revealed an enlarging heterogeneous, progressively enhancing solid and cystic mass in the anterior segment of the right hepatic lobe (Couinaud's segments V and VIII). The mass measured $8.9 \times 8.7 \times 7.5 \mathrm{~cm}$ and displaced hepatic and portal veins. As a result of the increasing size of this partially cystic and solid liver mass over the course of 18 months, surgical excision of the mass was recommended.

In July 2008, the patient underwent a right trisegmentectomy and cholecystectomy. The pathological report identified a $19 \times 17 \times 11 \mathrm{~cm}$ resected hepatic lobe weighing $1,006 \mathrm{~g}$, with a $9 \times 9 \times 7.5 \mathrm{~cm}$, well-circumscribed, pink-yellow-tan, gelatinous mass, with a $1 \mathrm{~cm}$ clear surgical margin. The mass was cystic in the central portion and contained $30 \mathrm{ml}$ of clear, yellowish fluid. Histologically, the mass consisted of rare benign dilated bile ducts ( $\underline{\text { fig. }}$ ) corresponding to the cystic areas noted grossly, as well as myxoid stroma with spindle cells showing smooth muscle differentiation confirmed by positive staining for vimentin, smooth muscle actin and desmin. The mass' fibrous capsule showed bile duct proliferation at the edge with a sharp transition to normal liver parenchyma. CD34 and hormone receptor studies were negative, thus excluding solitary fibrous tumor and angiomyxoma. The patient's hospital course was uneventful and she was discharged home on postoperative day five.

\section{Discussion}

MHL is a benign lesion of the liver representing the second most common pediatric liver tumor (after hepatoblastoma) and constitutes $8 \%$ of all pediatric tumors. Eighty percent of the lesions are found by the age of two and the remainder are identified by five years of age with the male to female ratio being 2:1 [2]. Intrauterine MHL cases have been documented in several reports. Laberge et al. [4] reviewed twelve cases with eight fetuses surviving to term after being diagnosed ultrasonographically in the third trimester. 
Tumor resection in these eight cases occurred in the first three months of life. In the four lethal intrauterine MHL cases, Laberge et al. attributed the fetal demise to mass effects of the tumor causing compression of the heart, kidneys and umbilical veins [4]. Although the majority of MHL cases reported are in the pediatric population, 30 cases have been reported in the English and Japanese language literature in adults ranging in age from 19 to 69 years (table 1) [5-7]. Of these 30 cases, 22 (73\%) have been reported in females and only $8(27 \%)$ in males, with an average age of 40 and 57 , respectively. Thus, males are more often affected in the pediatric population while females are more often affected in the adult population.

The clinical presentation of MHL appears to depend on the age of the patient. Most pediatric patients present with painless abdominal enlargement normally appreciated by the parent. If the mass becomes significantly large or liver function becomes compromised, ascites and jaundice may ensue. In severe cases there may be compression of the diaphragm and lungs causing respiratory difficulties. Although the adult case presented here was asymptomatic, Cook et al. [2] reported on eight adult cases, seven of whom were symptomatic, with the presenting complaint being diffuse abdominal pain. In agreement with Cook et al., a retrospective analysis of 17 cases by Yesim et al. [8] report that 10 cases were symptomatic. In addition to diffuse abdominal pain, reported symptoms and signs include hepatomegaly, right hypochondriac pain and left upper quadrant pain.

Radiographic appearance of MHL represents a continuum from predominantly cystic to mostly solid tumors. In pediatric patients the literature is unclear in regards to the cystic or solid nature of MHL. Early studies by Stocker and Ishak [9] describe nearly all pediatric cases as having cysts, however Chau et al. [10] reported that children more commonly have solid lesions. Interestingly, Chau et al. have also reported that adults have predominantly cystic lesions [10] while Hernandez et al. [5] have published that both pediatric and adult cases are primarily cystic. In the adult population, when solely cystic or solid tumors are present, females were more likely to have a cystic tumor, while males showed no predilection. When both cystic and solid portions of MHL were encountered, females were predominantly affected.

Location of the tumor in the liver appears to be different between pediatric and adult patients. MHL in children is primarily localized to the right lobe [10], while in adults, 12 cases (40\%) were localized to the left lobe, $12(40 \%)$ to the right lobe and in $6(20 \%)$ tumors crossed into both lobes. Interestingly, adult males have a predilection for the right lobe of the liver, while females primarily have MHL in the left lobe. In situations when MHL was found in both lobes, females were more commonly affected than males.

Ultrasonography is typically the initial evaluation technique for suspected hepatic masses since it is readily accessible, can quickly delineate the cystic or solid nature of the lesion, and provides an estimate of mass size. CT imaging and/or MRI typically follow ultrasonography, particularly in preparation for surgical resection of the tumor. Ultrasonographic images of MHL show anechoic cystic regions with intervening thin septations, and axial imaging is helpful to further demonstrate the cystic or solid nature of MHL [11]. On contrast-enhanced CT the solid component of MHL will enhance while the cystic portion will not [11]. MRI shows a characteristic low signal intensity on T1-weighted images and high intensity imaging on T2-weighted imaging [12] (table 2).

Even though multiple radiographic imaging techniques may be employed in the evaluation of a hepatic mass, imaging results for MHL are nonspecific. The differential diagnosis for predominantly cystic lesions includes: simple hepatic cyst, hydatid cyst, 
subcapsular pseudocyst, biliary cystadenocarcinoma, and cystic metastasis. Mortele and Ros [13] depict the radiographic nature of a variety of cystic liver lesions, particularly noting CT and MRI subtleties that may be helpful in diagnosis. In the case of a solid lesion, focal nodular hyperplasia, hepatic adenoma, cavernous hemangioma, angiomyolipoma, solitary hamartoma, and hepatocellular carcinoma are among the more common lesions to consider. A solid MHL may present as a hypovascularized lesion on angiography [13], but this is a nonspecific finding and not helpful in differentiating from the aforementioned lesions. In the present case fine needle biopsy did not reveal a definitive diagnosis, necessitating surgical excision of the lesion.

Recent cytogenetic studies have shown MHL to involve rearrangements at chromosome 19. Analysis of eight MHL cases revealed the following genetic abnormalities: three involving $\mathrm{t}(11 ; 19)(\mathrm{q} 13 ; \mathrm{q} 13.4)$, and single cases of $\mathrm{t}(11 ; 19)(\mathrm{q} 13 ; \mathrm{q} 13.3)$, $\mathrm{t}(15 ; 19)(\mathrm{q} 15 ; \mathrm{q} 13.4)$, a rearrangement of 11q2, 17q11 and 19q13.3, del(19)(q13.1q13.4) and $t(11 ; 19)(q 11 ; q 13.4)$ [14]. A significant finding is the similar genetic profile MHL shares with undifferentiated embryonal sarcoma (UES). UES is a rare pediatric malignancy composed of a sarcomatous mesenchymal component, as well as having hypercellular stroma, nuclear pleomorphism and mitotic activity, interspersed with benign branching ductal structures [3]. Over the past few years a number of cases of UES have developed within MHL, displaying similar genetic rearrangements in the region of $19 q 13.4[3,15,16]$. These include two cases of (19)(q13.4) and one case showing $t(11 ; 19)$ (q11;q13.3-13.4), nearly identical to genetic rearrangements seen in previous solitary cases of MHL [16]. Rajaram et al. [14] have postulated that the $t(11 ; 19)$ translocation is likely related to the development of solitary cases of MHL, but for progression of MHL to UES additional genetic alterations at other loci are required.

In addition to cytogenetic similarities, histological resemblance provides a potential link for progression from a benign MHL lesion to malignant UES. In cases described by Lauwers et al. [3] and Begueret et al. [15], UES regions were separated from MHL areas by transitional zones containing a histological mixture of the two entities. On further analysis, similar immunohistochemical characteristics and DNA ploidy of the three zones led Begueret et al. to conclude a coexistence of a well-differentiated phenotype (MHL) and a sarcomatous region (UES), separated by a zone containing a mixture of the two entities [15].

Hepatic angiosarcoma is the most common primary sarcoma of the liver and a solo case was recently reported to arise from an adult MHL [6]. Although hepatic angiosarcoma is usually associated with thorotrast, vinyl chloride and arsenic exposure, $\mathrm{Li}$ et al. [6] report a case of a woman with no prior carcinogen exposure presenting with an angiosarcoma arising from an untreated, previously diagnosed MHL. Though not direct evidence of MHL malignant transformation to angiosarcoma, the premalignant potential of MHL should be expanded to include UES and angiosarcoma, particularly when MHL is encountered in adult patients.

Although less invasive laparoscopic techniques are desirable, anatomic or nonanatomic resection with a negative margin is the standard treatment. The potential for massive tumor growth and possible degeneration to UES and/or angiosarcoma provides additional support against cautious clinical monitoring if the patient is a reasonable surgical candidate. Enlarging solid or cystic neoplasms of the liver that cannot be accurately diagnosed with radiographic imaging and histology should be considered for en bloc surgical resection with negative surgical margins. 


\section{Conclusion}

MHL in adults is a rare and potentially premalignant lesion that presents as a solid/cystic neoplasm. Symptoms are typically nonspecific, though abdominal pain is the most common. Laboratory results are noncontributory and radiographic imaging is variable and inconclusive. Needle biopsy is rarely diagnostic and surgical excision of symptomatic or enlarging lesions is recommended to exclude the possibility of malignancy and to establish a further diagnosis. The emerging literature suggests a relationship between MHL and UES in regards to histological similarities and cytogenetic analysis, and supports a recommendation for aggressive surgical management when feasible.

Table 1. Cases of adult MHL

\begin{tabular}{|c|c|c|c|c|c|c|}
\hline Number & Reference & Age & Gender & Liver lobe & Size & $\begin{array}{l}\text { Gross } \\
\text { appearance }\end{array}$ \\
\hline 1 & Yamamura [17] & 22 & $\mathrm{~F}$ & both & not described & cystic \\
\hline 2 & Grases [18] & 19 & $\mathrm{~F}$ & left & $24 \times 19 \times 8 \mathrm{~cm}$ & cystic \\
\hline 3 & Dooley [19] & 21 & $\mathrm{~F}$ & right & $26 \times 20 \times 13 \mathrm{~cm}$ & cystic \\
\hline 4 & Kurokawa [20] & 43 & $\mathrm{~F}$ & left & $22 \times 15 \times 10 \mathrm{~cm}$ & solid \\
\hline 5 & Ishizuka [21] & 59 & M & not described & $30 \times 28 \times 12 \mathrm{~cm}$ & cystic \\
\hline 6 & Kawakami [22] & 67 & M & right & not described & cystic \\
\hline 7 & Jennings [23] & 32 & $\mathrm{~F}$ & left & $14 \times 11 \mathrm{~cm}$ & cystic \\
\hline 8 & Kato [24] & 66 & M & left & not described & solid \\
\hline 9 & Gutierrez [25] & 30 & $\mathrm{~F}$ & both & $18 \mathrm{~cm}$ & both \\
\hline 10 & Gramlich [26] & 28 & $\mathrm{~F}$ & right & $30 \times 20 \times 14 \mathrm{~cm}$ & both \\
\hline 11 & Alanen [27] & 20 & $\mathrm{~F}$ & left & $6 \times 8 \mathrm{~cm}$ & both \\
\hline 12 & Ito [28] & 43 & $\mathrm{~F}$ & both & $16 \times 16 \times 7.7 \mathrm{~cm}$ & cystic \\
\hline 13 & Urabe [29] & 39 & $\mathrm{~F}$ & left & $1.2 \mathrm{~cm}$ & solid \\
\hline 14 & Drachenberg [30] & 69 & $\mathrm{~F}$ & left & $26 \times 20 \times 11.5 \mathrm{~cm}$ & both \\
\hline 15 & Wada [31] & 62 & M & left & $6 \times 6 \times 4.5 \mathrm{~cm}$ & solid \\
\hline 16 & Chau [10] & 53 & M & right & $20 \times 14 \times 10 \mathrm{~cm}$ & cystic \\
\hline 17 & Megremis [32] & 56 & $\mathrm{~F}$ & both & $7.5 \mathrm{~cm}$ & cystic \\
\hline 18 & Chung [33] & 57 & $\mathrm{~F}$ & left & $6 \times 4 \times 3.5 \mathrm{~cm}$ & solid \\
\hline 19 & Papastratis [34] & 21 & $\mathrm{~F}$ & right & $17 \times 10 \mathrm{~cm}$ & cystic \\
\hline 20 & Cook [2] & 46 & $\mathrm{~F}$ & right & $6 \times 4 \times 5 \mathrm{~cm}$ & cystic \\
\hline 21 & Cook [2] & 66 & $\mathrm{~F}$ & right & $5 \times 4 \times 2 \mathrm{~cm}$ & cystic \\
\hline 22 & Cook [2] & 63 & $\mathrm{~F}$ & left & $11 \times 16 \times 24 \mathrm{~cm}$ & solid \\
\hline 23 & Brkic [35] & 38 & M & right & $8 \times 5 \mathrm{~cm}$ & solid \\
\hline 24 & $\operatorname{Kim}[36]$ & - & M & right & $5 \mathrm{~cm}$ & both \\
\hline 25 & Yesim [8] & 54 & $\mathrm{~F}$ & left & $2.5 \times 2.5 \times 1.5 \mathrm{~cm}$ & cystic \\
\hline 26 & Yesim [8] & 51 & $\mathrm{~F}$ & right & $6 \times 7 \times 8 \mathrm{~cm}$ & cystic \\
\hline 27 & Ayadi-Kaddour [37] & 21 & $\mathrm{~F}$ & left & $11 \times 5 \mathrm{~cm}$ & cystic \\
\hline 28 & Hernandez [5] & 51 & M & right & $19 \times 13 \mathrm{~cm}$ & solid \\
\hline 29 & $\mathrm{Li}[6]$ & 33 & $\mathrm{~F}$ & both & not described & angiosarcoma \\
\hline 30 & Mori [7] & 36 & $\mathrm{~F}$ & right & $20 \times 15 \times 10 \mathrm{~cm}$ & cystic \\
\hline 31 & Current case & 53 & $\mathrm{~F}$ & both & $9 \times 9 \times 7.5 \mathrm{~cm}$ & both \\
\hline
\end{tabular}


Table 2. Radiographic appearance of MHL [11-13]

\begin{tabular}{ll}
\hline Imaging modality & Radiographic appearance \\
\hline Ultrasound & Anechoic, multilobular mass with thin septations. \\
\hline Computed tomography & $\begin{array}{l}\text { Appearance ranges from a multilocular cystic mass with solid septa to } \\
\text { multiple small cysts in a solid mass. Contrast administration enhances the } \\
\text { solid component without a change in the cystic portion. }\end{array}$ \\
\hline $\begin{array}{l}\text { Magnetic resonance } \\
\text { imaging }\end{array}$ & $\begin{array}{l}\text { The cystic component will show low signal intensity on T1-weighted images } \\
\text { and high intensity on T2-weighted images. Solid component will depict low } \\
\text { intensity on T1-weighted images due to fibrosis. }\end{array}$ \\
\hline Angiography & $\begin{array}{l}\text { Cystic masses appear completely avascular due to displacement of major } \\
\text { vessels. Predominantly solid lesions are hypovascular with small tumor vessels } \\
\text { correlating to septa vascularization surrounding scattered cystic regions. }\end{array}$ \\
\hline
\end{tabular}

Fig. 1. Noncontrast CT scan from February 2007 demonstrating a $5.3 \mathrm{~cm}$ septate hypodense cystic lesion in the right lobe of the liver.

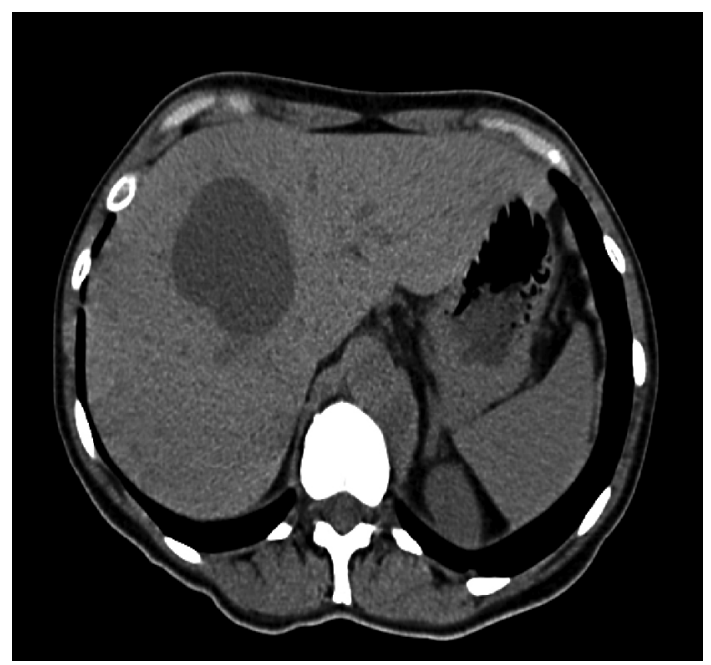




\begin{tabular}{r|l|l|l}
$\begin{array}{r}\text { Case Reports in } \\
\text { Gastroenterology }\end{array}$ & $\begin{array}{l}\text { Case Rep Gastroenterol 2010;4:84-92 } \\
\text { D0I: 10.1159/000260183 }\end{array}$ & Published online: March 13, 2010 & $\begin{array}{l}\text { O 2010 S. Karger AG, Basel } \\
\text { ISSN 1662-0631 } \\
\text { www.karger.com/crg }\end{array}$ \\
\hline
\end{tabular}

Fig. 2. Repeat abdominal CT scan in July 2008 revealing an enlarging heterogeneous, progressively enhancing solid and cystic mass in the anterior segment of the right hepatic lobe (Couinaud's segments V and VIII). The mass measures $8.9 \times 8.7 \times 7.5 \mathrm{~cm}$ and displaces hepatic and portal veins.

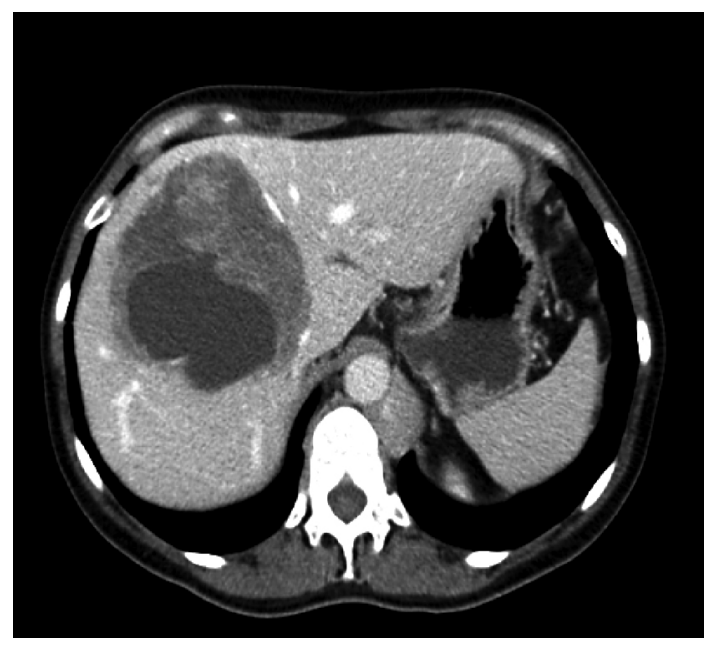

Fig. 3. Microscopic image. The liver mass consists of rare benign dilated bile ducts corresponding to the cystic areas. The cytologically benign stroma is extremely myxoid with spindle cells. The mass had a fibrous capsule with bile duct proliferation at the edge with a sharp transition into the normal liver tissue (arrow), diagnostic of mesenchymal hamartoma. 10×, HE stain.

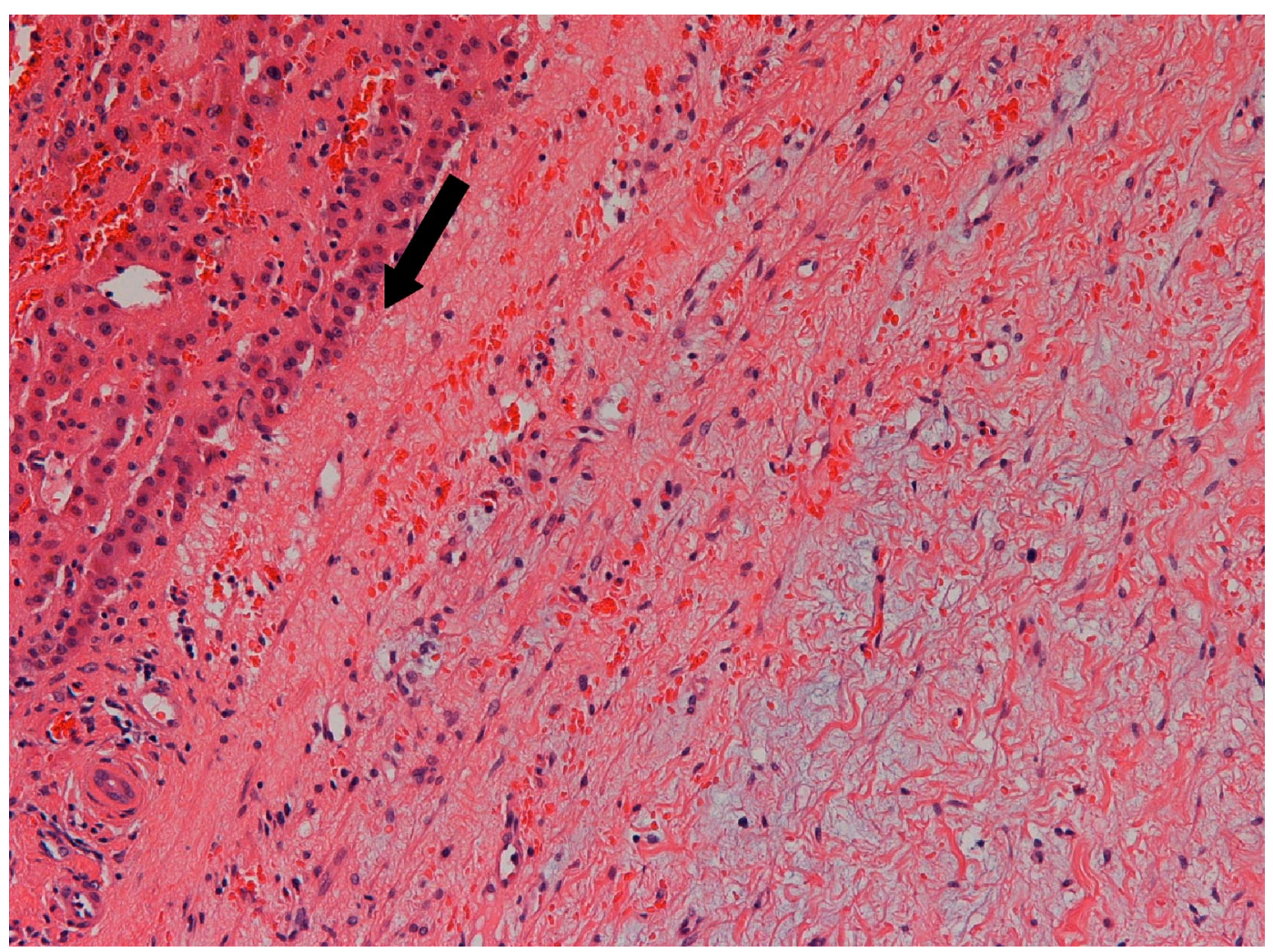




\section{References}

1 Edmondson HA: Differential diagnosis of tumors and tumor-like lesions of liver in infancy and childhood. Am J Dis Child 1956;91:168-186.

2 Cook JR, Pfeifer JD, Dehner LP: Mesenchymal hamartoma of the liver in the adult: Association with distinct clinical features and histological changes. Hum Pathol 2002;33:893-898.

3 Lauwers GY, Grant LD, Donnelly WH, Meloni AM, Foss RM, Sanberg AA, Langham MR Jr: Hepatic undifferentiated (embryonal) sarcoma arising in a mesenchymal hamartoma. Am J Surg Pathol 1997;21:1248-1254.

4 Laberge JM, Patenaude Y, Desilets V, Cartier L, Khalife S, Jutras L, Chen MF: Large hepatic mesenchymal hamartoma leading to midtrimester fetal demise. Fetal Diagn Ther 2005;20:141-145.

5 Hernandez JC, Alfonso C, Gonzalez L, Samada M, Ramos L, Cepero-Valdez M, Antonio Abdo A, Gomez F, Castellanos R, Lopez O, Ugarte JC, Jordan J: Solid mesenchymal hamartoma in an adult: A case report. J Clin Pathol 2006;59:542545.

6 Li O, Wang J, Sun Y, Cui Y, Hao X: Hepatic angiosarcoma arising in an adult mesenchymal hamartoma. Int Semin Surg Oncol 2007;4:3.

7 Mori R, Morioka D, Morioka K, Ueda M, Sugita M, Takeda K, Matsuo K, Tanaka K, Endo I, Sekido H, Togo S, Shimada H: Giant mesenchymal hamartoma of the liver in an adult. J Hepatobiliary Pancreat Surg 2008;15:667-669.

8 Yesim G, Gupse T, Zafer U, Ahmet A: Mesenchymal hamartoma of the liver in adulthood: immunohistochemical profiles, clinical and histopathological features in two patients. J Hepatobiliary Pancreat Surg 2005;12:502-507.

9 Stocker J, Ishak KG: Mesenchymal hamartoma of the liver: report of 30 cases and review of the literature. Pediatr Pathol 1983;1:245-267.

10 Chau KY, Ho JW, Wu PC, Yuen WK: Mesenchymal hamartoma of liver in a man: Comparison with cases in infants. J Clin Pathol 1994;47:864-866.

11 Donovan TA, Wolverson MK, DeMello D, Craddock T, Silberstein M: Multicystic hepatic mesenchymal hamartoma of childhood: CT and US characteristics. Pediatr Radiol 1981;11:163-165.

12 Cetin M, Demirpolat G, Elmas N, Yuce G, Cetingul N, Balik E: Stromal predominant type mesenchymal hamartoma of liver: $\mathrm{CT}$ and MR features. Comput Med Imaging Graph 2002;26:167-169.

13 Mortele KJ, Ros PR: Cystic focal liver lesions in the adult: differential CT and MRI imaging features. Radiographics 2001;21:895-910.

14 Rajaram V, Knezevich S, Bove KE, Perry A, Pfeifer JD: DNA sequence of the translocation breakpoints in undifferentiated embryonal sarcoma arising in mesenchymal hamartoma of the liver harboring the $t(11 ; 19)(\mathrm{q} 11 ; \mathrm{q} 13.4)$ translocation. Genes Chromosomes Cancer 2007;46:508-513.

15 Begueret H, Trouette H, Vielh P, Laurent C, MacGrogan G, Delsol M, Belleannee G, Masson B, De Mascarel A: Hepatic undifferentiated embryonal sarcoma: malignant evolution of mesenchymal hamartoma? Study of one case with immunohistochemical and flow cytometric emphasis. J Hepatol 2001;34:178-179.

16 O'Sullivan MJ, Swanson PE, Knoll J, Taboada EM, Dehner LP: Undifferentiated embryonal sarcoma with unusual features arising within mesenchymal hamartoma of the liver; report of a case and review of the literature. Pediatr Dev Pathol 2001;4:482-489.

17 Yamamura T, Kameyama Y, Hoshino T, Hayakawa K: A case of mesenchymal hamartoma of the liver coexisting with congenital dilatation of the bile duct. J Jpn Soc Clin Surg 1976;37:862.

18 Grases PJ, Matos-Villalobos M, Arcia-Romero F, Lecuna-Torres V: Mesenchymal hamartoma of the liver. Gastroenterology 1979;76:1466-1469.

19 Dooley JS, Li AKC, Scheuer PJ, Hobbs KEF, Sherlock S: A giant cystic mesenchymal hamartoma of the liver: Diagnosis, management, and study of cyst fluid. Gastroenterology 1983;85:958-961.

20 Kurokawa H, Sasaki K, Kamita N, Yamaguchi Y, Kikuchi Y, Kojimahara M: A case of huge hepatic hamartoma. Jpn J Clin Radiol 1984;29:901-904.

21 Ishizuka M, Miyashita M, Takahama M: A case of giant mesenchymal hamartoma of the liver found in an adult. Proc Jpn Cancer Assoc 1985;44:490. 
22 Kawakami A, Fujii H, Ono Y: A case of hepatic mesenchymal hamartoma in an adult. Nippon Act Radiol 1986;46:1073.

23 Jennings CM, Merrill CR, Slater DN: Case report: The computed tomographic appearances of benign hepatic hamartoma. Clin Radiol 1987;38:103-104.

24 Kato Y, Yokozaki H: Hepatic mass (mesenchymal hamartoma). J Hiroshima Med Assoc 1988;41:143.

25 Gutierrez OH, Burgener FA: Mesenchymal hamartoma of the liver in an adult: radiologic diagnosis. Gastrointest Radiol 1988;13:341-344.

26 Gramlich TL, Killough BW, Garvin AJ: Mesenchymal hamartoma of the liver: Report of a case in a 28-year-old. Hum Pathol 1988;19:991-992.

27 Alanen A, Katevuo K, Toikkanen S: A non-cystic mesenchymal hamartoma of the liver - an unusual case of an unusual entity. Case report and review of the literature. Bildgebung 1989;56:181-184.

28 Ito Y, Seto Y, Okano H, Ueda T, Imanishi H, Nakamura K, Nakajima T, Okanoue $\mathrm{T}$ : A case of mesenchymal hamartoma of the liver in adult woman with pancreas divisum and marked delay in ICG plasma clearance. Nippon Shokakibyo Gakkai Zasshi 1989;86:1534-1539.

29 Urabe S, Furukawa M, Nakata T: A case of mesenchymal hamartoma of the liver in an adult. Jpn J Gastroenterol Surg 1990;23:664.

30 Drachenberg CB, Papadimitriou JC, Rivero MA, Wood C: Adult mesenchymal hamartoma of the liver: Report of a case with light microscopic, FNA cytology, immunohistochemistry, and ultrastructural studies and review of the literature. Mod Pathol 1991;4:392-395.

31 Wada M, Ohashi E, Jin H, Nishikawa M, Shintani S, Yamashita M, Kano M, Yamanaka N, Nishigami T, Shimoyama T: Mesenchymal hamartoma of the liver: report of an adult case and review of the literature. Inter Med 1992;31:1370-1375.

32 Megremis S, Sfakianaki E, Voludaki A, Chroniaris N: The ultrasonographic appearance of a cystic mesenchymal hamartoma of the liver observed in a middleaged woman. J Clin Ultrasound 1994;22:338-341.

33 Chung JH, Cho KJ, Choi DW, Lee BH, Chi JG: Solid mesenchymal hamartoma of the liver in adult. J Korean Med Sci 1999;14:335-337.

34 Papastratis G, Margaris H, Zografos GN, Korkolis D, Mannika Z: Mesenchymal hamartoma of the liver in an adult: a review of the literature. Int J Clin Pract 2000;54:552-554.

35 Brkic T, Hrstic I, Vucelic B, Jakic-Razumovic J, Skegro M, Romic B, CukovicCavka S, Pulanic R, Ostojic R: Benign mesenchymal liver hamartoma in an adult male: a case report and review of the literature. Acta Med Austriaca 2003;30:134137.

36 Kim MH, Choi MS, Lee JH, Koh KC, Paik SW, Yoo BC, Jung JH, Choi SC, Kim DH, Lee H, Song BG, Rhee JC, Park CG: Mesenchymal hamartoma of the liver in adults. Taehan Kan Hakhoe Chi 2003;9:31-34.

37 Ayadi-Kaddour A, Saiji E, Ben Slama S, Chelly-Ennaiffer I, Lahmar-Boufaroua A, Goutallier-Ben Fadhel C, Ben Sassi L, Khalfallah MT, Mzabi-Regaya S: Hepatic mesenchymal hamartoma in adulthood: a case report with literature review. Tunis Med 2006;84:263-265.

All the authors listed declare that there are no conflicts of interest and that no financial sponsorship was accepted in producing and presenting this article. Each of the authors listed is in agreement with the content of the paper. 